\title{
TISSUE VIABILITY: THE ROLE OF WOUND BED PREPARATION IN WOUND MANAGEMENT
}

\author{
D Wilson, Tissue Viability/Infection Control Nurse \\ Furness General Hospital
}

Tissue Viability (TV) is often an under-resourced and underused facility, and sometimes confusion arises between staff and TV practitioners about when to contact the department for advice. The TV nurse (TVN) is a resource to be used when general wound-care products or treatments have failed and more specific advice is needed. It is hoped there will be a more formal referral system in place very soon, which should aid staff in understanding the patients who require referral for wound advice. As the role develops it is anticipated that there will be more training sessions for staff to enable them to understand wound healing and how to use products effectively throughout the process of wound healing.

The healing of chronic wounds is still not fully understood $^{(1)}$, yet the cost to the health community can be immense in terms of both finance and morbidity. The concept of wound bed preparation has provided a rationale for nursing and medical staff to follow in preparing the wound bed for healing.

It is now 40 years since Winter published his findings regarding moist wound healing ${ }^{(2)}$. In some respects very little has been learned since then - for example wet to dry debridement continues to be recommended ${ }^{(3)}$. The pain and suffering experienced by patients undergoing such treatments cannot easily be measured. Patient satisfaction surveys are rare in randomized controlled trials, but in wound care, trials should be a consideration. In the twenty-first century there are treatments available which have the same effect without the cost, in terms of pain and suffering, to the patient.

Falanga $^{(4)}$ and Sibbald ${ }^{(5)}$ advocate wound bed preparation. This concept challenges out-of-date misconceptions of wound care. Falanga proposes a framework and reports measurable healing outcomes for chronic wounds. Chronic wounds do not follow precisely the phases of healing described so frequently in the literature ${ }^{(4)}$. For the chronic wound to heal effectively the local conditions in the wound bed have to be prepared before the healing rate can be measured. It is generally accepted that there are three main factors in the local wound environment which require attention:

- control of oedema

- reduction of the bacterial burden

- removal of dead tissue.

The chronic wound may display one or more of the problems listed. Getting the wound environment balanced is the key to success. This can be achieved by using modern dressings effectively. There are many products on the market, and no 'one size fits all'. The practitioner has to understand both the wound healing mechanism and how products are formulated, their action and the length of time they can be left in place, to gain optimum results in terms of wound healing and cost effectiveness. There is little point in recommending an expensive dressing if it will not do the job required.

\section{CONTROL OF OEDEMA}

This may be difficult to achieve immediately. It almost goes without saying that the underlying conditions should be treated first. Wounds on the lower leg in patients with heart failure are unlikely to heal or remain healed if the underlying heart condition is not considered and treated accordingly. Locally, within the wound bed environment, exudate can be managed with a variety of dressings: alginates, spun hydrocolloids or foams may be useful for light to heavy exudate. For heavily exuding wounds there is a variety of dressings that contain a gel to manage the situation; of course, more frequent dressing changes, with a less interactive dressing, may sometimes be the best approach. For problems with the lower leg, elevation should always be an early consideration to aid in the control of oedema. Compression in the form of bandaging or hosiery may also be a requirement.

\section{REDUCING THE BACTERIAL BURDEN}

This can be achieved within the wound environment by using medicated products such as iodine. Falanga ${ }^{(6)}$ has found cadoxemer iodine to be particularly effective as the bacteria are taken into the cadoxemer molecules and are not released back into the wound environment as the product degrades. Iodine comes in many formats, but the products, which are in a matrix, are generally easy and safe to use as the dosage is clearly stated on the product information. Solutions cannot be measured in terms of dosage to a wound, and are less stable and quickly ineffective in the presence of organic matter.

Silver is another product which is effective in reducing the bacterial burden. Again there are many products available on the market, each having a slightly different presentation and wear time. Silver has a bacteriostatic action on bacteria, thus reducing the bacteria's capability for replication.

Altering the bacterial environment locally can be more effective than systemically treating a patient. This is because the delivery of an antibiotic to the wound bed may be haphazard and penetration of the tissues is not guaranteed, particularly if there are circulatory problems. The use of broad spectrum and topical antibiotics is not recommended. 


\section{REMOVAL OF DEAD TISSUE}

This can be achieved in a variety of ways:

- surgical debridement

- sharp excision

- autolytic

- bio-surgery.

Surgical debridement is the quickest and in certain circumstances the only course of action to be taken, eg necrotising fasciitis, where removal of infected tissue is paramount. In some instances surgical debridement is not always undertaken as the patient may be an anaesthetic risk. Sharp debridement by an experienced practitioner would then be the treatment of choice.

Autolytic debridement is achieved by maintaining a moist wound environment, thereby allowing natural processes to separate necrotic and viable tissue. The use of hydrogels and hydrocolloids, though not necessarily together, will achieve the required outcome in most instances.

Larval therapy may also be used if the tissue requiring removal is hydrated enough. A wound containing large amounts of dry necrotic tissue is not suitable initially, but once softened, larvae may be a treatment option. Caution has to be taken when rehydrating eschar, as larvae do not survive if hydrogels have been used up to two days prior to application unless the wound is thoroughly irrigated before applying the larvae.

Wound bed preparation is not a single concept theory. It is a process of moving from the belief that any single intervention ought to work on patients on every occasion and heal them overnight to an understanding that there may not be a single intervention appropriate throughout the healing process. This should prove very useful in practice: not only may some wounds now heal but out-of-date misconceptions of wound care will be challenged. Significantly for budget holders, wound bed preparation promotes clinical cost effectiveness by focusing clinical decision-making on the correct treatment.

\section{CELLULITIS}

Cellulitis is a common reason for admission to hospital throughout the trust, and is an ideal condition to highlight the effective use of wound bed preparation. The care of patients with cellulitis is not standardized within the trust and guidelines vary between sites. It was with this background in mind that an audit was undertaken of patients admitted with cellulitis. The audit was designed to produce information about the length of hospital stay, antibiotics used, predisposition to recurrent cellulitis and presenting factors on admission.

The antibiotic guidelines at RLI include the recommendation that "support in the form of hosiery or even compression bandages accelerates healing and improves comfort'. These guidelines were written by Carol Magee (infection control nurse) and Drs Telford and Walmsley (consultant microbiologist and consultant physician) in 1998 and have been used on the RLI site since then. In the literature there are no published articles advocating the use of compression in the acute phase of cellulitis and most authors recommend removing compression on the grounds of pain.
This is of interest as when compression is used there is an acceleration of healing and earlier resolution of cellulitis, and patients rarely complain of increased pain. In fact, most patients report increased comfort with the use of compression, as the limb feels better supported.

A retrospective audit of patients admitted with cellulitis was conducted to assess whether the guidelines were being followed.

\section{RESULTS OF THE AUDIT}

Thirty-three patient records were reviewed. Length of admission was measured and varied from 0 to 28 days (average length of stay: nine days). The main presenting symptoms were red skin and oedema (Figure 1). Local heat, fever and 'flu-like symptoms were reported in fewer than half the cases. Pain was a significant factor in 13 cases.

Predisposing factors were more varied (Figure 2). A history of cellulitis was the main factor; existing wound and leg oedema were also relevant.

The antibiotics used were reasonably consistent across the trust (Figure 3). Although there is a recommendation for the use of compression within the audit only two patients received compression, one at RLI and one at FGH.

\section{DISCUSSION}

The results of the audit are interesting. They indicate a need for further effort to reduce the length of hospital stay for some patients, provide an environment for earlier resolution of cellulitis and form longterm strategies to reduce the number of people readmitted with cellulitis. This cannot be achieved in one study; I have proposed a trial in which we shall, initially, look at reducing oedema in patients by using short stretch compression bandaging. In measuring the reduction of oedema and earlier resolution of cellulitis patients should be discharged more quickly, providing benefits in terms of available bed days to the trust. The trial will take the form of a randomized controlled trial; research and ethical approval are being sought.

The study will have two groups. One will receive antibiotics and no compression, the other will receive antibiotics and compression using short stretch bandages whilst an inpatient.

There is an ethical dilemma for staff in that using compression without a Doppler assessment goes against national guidelines ${ }^{(7,8)}$. The value of such Doppler results is, however, questionable when the patient has oedema, as they may be incorrect ${ }^{(9)}$. A holistic assessment of the patient and pulse oximetry should give an adequate baseline to recommend compression. The use of short stretch bandaging also adds a safety factor, as this type of bandage has a high working/low resting pressure. The bandage forms an inelastic cuff around the lower leg compressing the calf muscle pump when standing or walking. A cohesive bandage presentation is to be recommended for the trial.

The benefit of using a short stretch bandage system is that there are only two layers to apply, which makes training of staff much easier. The bandage is applied in spiral form and is not applied in the same manner as a four-layer system; staff do not have to remember the different layers and their mode 


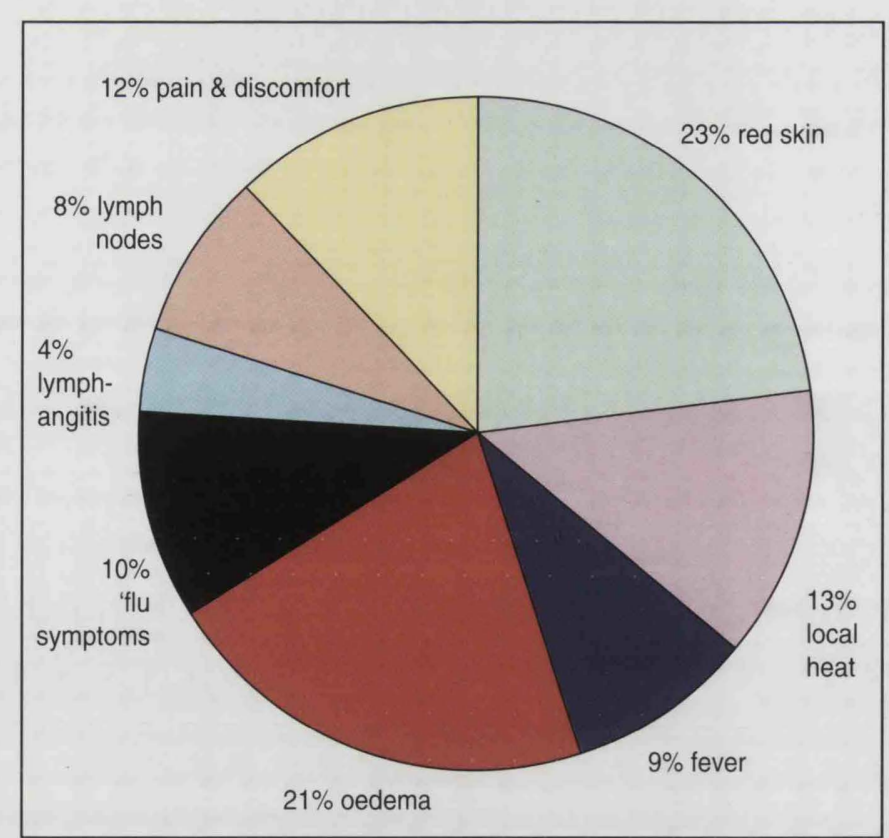

Figure 1 Symptoms

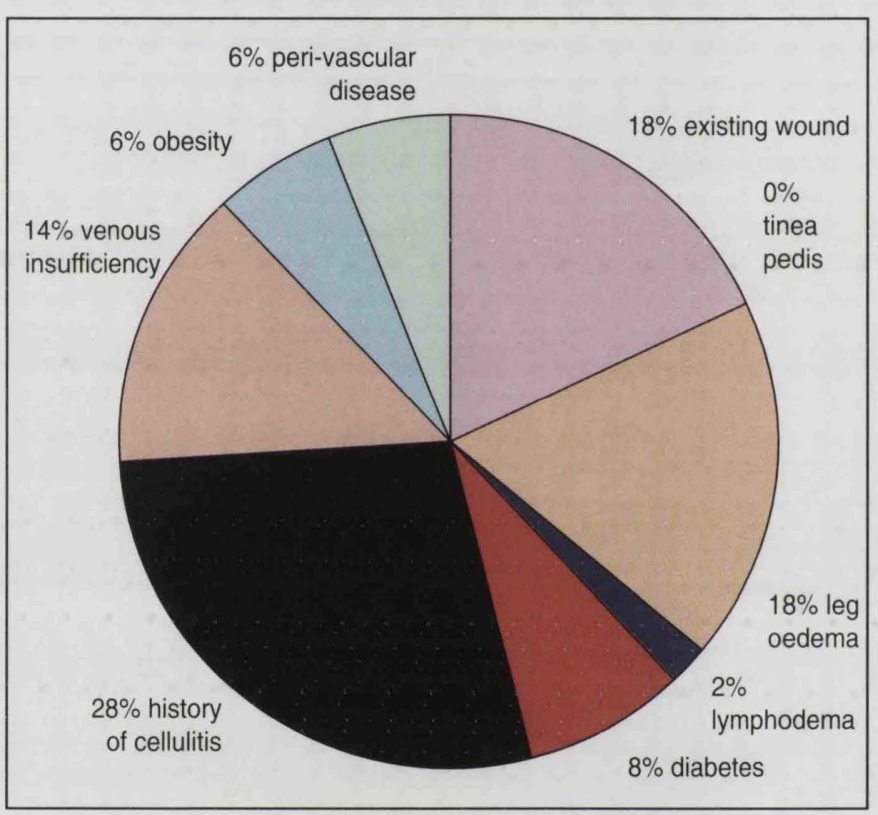

Figure 2 Pre-disposition

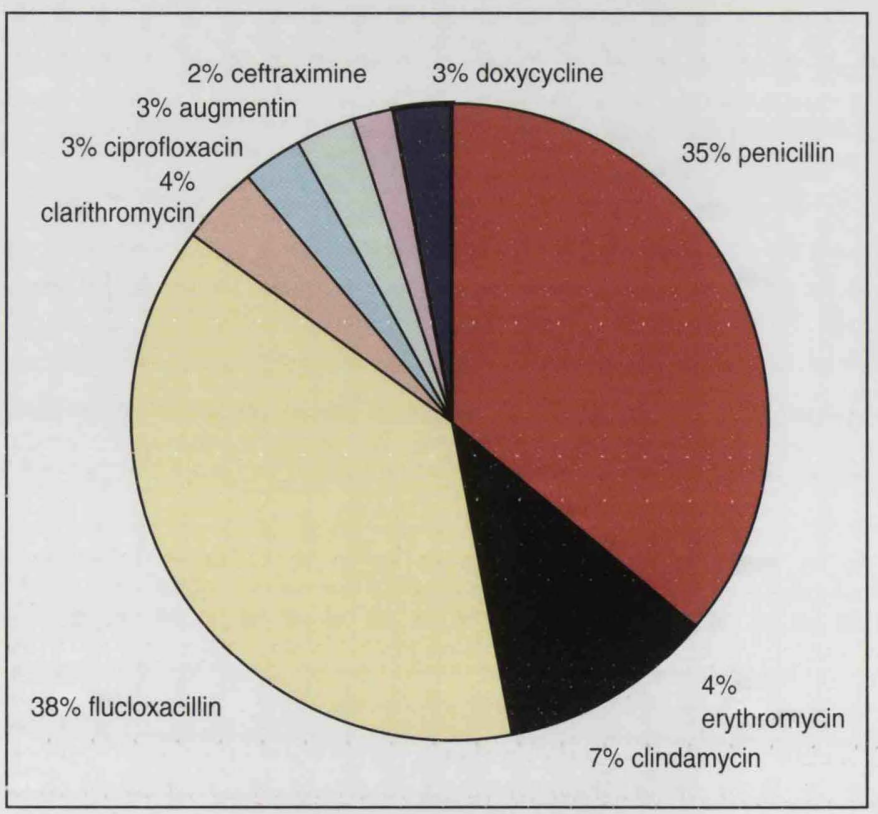

Figure 3 Antibiotic prescribing of application. An initial layer of cotton-wool bandage is applied and then the compression bandage is applied at full stretch with a $50 \%$ overlap from toe to knee. If the limb circumference is above $25 \mathrm{~cm}$ a second layer of bandage will be applied from ankle to knee.

In practice the results have been quicker resolution of cellulitis and reduction in the limb circumference, which has lead to earlier discharge of patients. Patients who have broken skin also benefit as the wounds heal more quickly. There is a need for further work in the community which must be addressed at a later date. The longterm management of patients who experience recurrent cellulitis needs to be addressed. This is work which can be carried out after the initial study.

\section{CONCLUSION}

Tissue viability is an exciting area of patient care which complements the multi-disciplinary team approach. The TVN should act as an advisor in difficult situations and take the role of innovator and educator in wound care practice, thereby enhancing the effectiveness of care received by patients and the knowledge and competence of staff.

\section{REFERENCES}

1 Collier M. Wound-bed preparation. Nursing Times Plus 2002;98,(2)

2 Winter G. Formation of the scab and the rate of epithelialisation of superficial wounds in the skin of the young domestic pig. Nature 1962;193:293-294

3 National Institute for Clinical Excellence (NICE) 2001 www.nice.org.uk

4 Falanga V. Classifications for wound bed preparation and stimulation of chronic wounds. Wound Repair and Generation 2000;8:5

5 Sibbald G. Preparing the wound bed - debridement, bacterial balance, and moisture balance. Ostomy Wound Management 2000;46

6 Falanga V. Iodine-containing pharmaceuticals: a reappraisal. Proceedings of the 6th European Conference on Advances in Wound Management 1997. London. Macmillan Magazines Ltd

7 Royal College of Nursing Guidelines (RCN). Clinical Practice Guidelines. The management of patients with venous leg ulcers. London: RCN 1998

8 Scottish Intercollegiate Guidelines Network (SIGN Publication No.26) Edinburgh: SIGN, 1998

9 Anderson I. The use of short stretch bandaging to control oedema. J Wound Care 1998;7(1):10-12

10 Vowden K, Vowden P. Doppler and the ABPI: how good is our understanding? J Wound Care 2001;10(6)

\section{FURTHER INFORMATION}

www.bu.edu/woundbiotech

www.Twhc.ca

www.Whru.co.uk 